\title{
Antibiotic resistance properties of uropathogenic Escherichia coli isolated from pregnant women with history of recurrent urinary tract infections
}

\author{
Asghar Habibi ${ }^{1}$ and Maryam Khayyat Khameneie ${ }^{2 *}$ \\ ${ }^{1}$ Department of Urology, Emam Reza Hospital, Birjand University of Medical Sciences, Birjand, ${ }^{2}$ Department of Gynecology, \\ Imam Reza Hospital, AJA University of Medical Sciences, Tehran, Iran
}

*For correspondence: Email: dr.khameneie@yahoo.com; Tel: +989121723880

\begin{abstract}
Purpose: To investigate the antibiotic resistance pattern of uropathogenic Escherichia coli (UPEC) strains isolated from pregnant women with history of recurrent urinary tract infections (RUTIS) and healthy pregnant women.

Methods: A total of 485 high vaginal swab specimens were collected from pregnant women with history of RUTIs and healthy ones. Samples were cultured and those that were E. coli positive were analyzed by polymerase chain reaction (PCR) and disk diffusion tests.

Results: Twenty eight out of 445 (6.29\%) samples were positive for E. coli. Prevalence of E. coli in pregnant women with history of RUTIs and healthy ones were 9 and $4.08 \%$, respectively. Women older than 40 years were the most commonly infected. UPEC strains showed the highest levels of resistance against ampicillin (96.42\%), tetracycline (85.71\%), amikacin (71.42\%), ciprofloxacin (67.85\%) and gentamycin (58.71\%).

Conclusion: RUTIs and pregnancy in old age are two important risk factors for transmission of UPEC strains from the urinary tract into the vagina. Primary treatment of infected pregnant women with imipenem, mezlocillin and nitrofurantoin would be effective for the prevention and management of vaginal infections in pregnant women.
\end{abstract}

Keywords: Uropathogenic Escherichia coli, Recurrent urinary tract infections, Antibiotic resistance, Pregnant women

Tropical Journal of Pharmaceutical Research is indexed by Science Citation Index (SciSearch), Scopus, International Pharmaceutical Abstract, Chemical Abstracts, Embase, Index Copernicus, EBSCO, African Index Medicus, JournalSeek, Journal Citation Reports/Science Edition, Directory of Open Access Journals (DOAJ), African Journal Online, Bioline International, Open-J-Gate and Pharmacy Abstracts

\section{INTRODUCTION}

Pregnancy causes numerous changes in the woman's body. During pregnancy, the level of body immune system is reduced. Hormonal and mechanical changes increase the risk of urinary stasis and vesicoureteral reflux. These changes, along with an already short urethra and difficulty with hygiene due to a distended pregnant belly, increase the frequency of urinary tract infections (UTIs) in pregnant women [1]. Indeed, UTIs are among the most common bacterial infections during pregnancy [1]. Urinary tract infections (UTIs) are among the most common bacterial infections diseases in human [2-4]. UTIs account for more than 8 million referrals to hospitals, 1.5 million hospitalization, and 300,000 severe clinical syndromes in the United States annually $[2,5]$. UTIs is an important cause of mortality and morbidity $[6,7]$.

Uropathogenic Escherichia coli (UPEC) strains are the most prevalent causes of UTIs around the world $[3,8]$. Based on the hypothesis, UPEC 
strains can transfer from the urinary tract of pregnant women who have low levels of immunity into the reproductive system $[9,10]$. This maybe more common in pregnant women with history of recurrent UTIs (EUTIs). Association of bacterial vaginosis and UTIs in pregnant women have also been reported previously [10-12].

In an overview, treatment of the cases of UTIs and vaginal infections were carried out by antibiotic therapy, but high prevalence of antibiotic resistance in the UPEC strains of clinical infections, with the attenelant prolonged severe diseases, $[3,13,14]$. According to recent epidemiological studies, UPEC strains showed a high prevalence of resistance (50-100 \%) against commonly used antibiotics, including tetracycline, cefotaxime, gentamycin, ampicillin, amikacin, ciprofloxacin (5 $\mu \mathrm{g} / \mathrm{disk})$, cotrimoxazole, norfloxacin and cephalothin $[3,13,14]$. with to the mutable status of the antibiotic resistance in UPEC strains of clinical samples in cases of pregnant women which are of high clinical importance, the distribution of antibiotic resistance pattern should be evaluated.

Due to the uncertain status of UPEC strains in pregnant women, the present study was carried out to investigate antibiotic resistance pattern of the UPEC strains isolated from pregnant women with history of RUTIs.

\section{EXPERIMENTAL}

\section{Ethical issues}

This work was approved by the ethical committee of Department of Infectious Diseases, College of Medicine, AJA University of Medical Sciences, Tehran, Iran (ref no. 20275 MD). Written informed consent was obtained from the patients or a close relative. All stages of sampling were done in such a way as to minimize pain and harm to the patients and international guidelines for human studies were followed [15].

\section{Study population and samples collection}

Total of 240 high vaginal swab specimens were collected from pregnant women with history of RUTIs from March to December 2015. Besides, 245 high vaginal swabs were also taken from healthy pregnant women who were referred to the Obstetrics and Gynecology Centers, Iran for routine pregnancy checks. Specimens were collected from the ventral fornix without any contact with urine and external parts of reproductive system, using speculum and commercial sterile cotton-tipped swabs. All specimens were collected by an expert midwife.

\section{Escherichia coli identification}

All samples were transferred to the laboratory in a cooler with ice packs. A, $3 \mathrm{~mL}$ of each specimen was blended with $225 \mathrm{~mL}$ of Lactose Broth (LB, Merck, Germany) for 2 min at normal speed, using a Stomacher lab blender and incubated at $37^{\circ} \mathrm{C}$ for $24 \mathrm{~h}$. One milliliter sample of the nutrient broth culture was mixed with $9 \mathrm{~mL}$ of MacConkey broth (MC broth, Merck, Germany) and further incubated at $37^{\circ} \mathrm{C}$ for 24 h. One loop of each tube was streaked on MacConkey agar (MC agar, Merck, Germany). A typical colony of $E$. coli was streaked on Eosin Methylene Blue agar (EMB agar, Merck, Germany) plates and incubated at $37^{\circ} \mathrm{C}$ for $24 \mathrm{~h}$. A green metallic colonies from each plate with typical E. coli morphology was selected and examined by biochemical tests, including hydrogen sulfide, citrate, urease, methyl red (MR), Voges-Proskauer (VP), Triple sugar iron agar (TSI agar) and indole.

\section{PCR confirmation of Escherichia coli strains}

Total genomic DNA was extracted from the bacterial colonies using the DNA purification kit (Fermentas, Germany) according to the instructions. The colonies were confirmed using PCR method [16]. The $10 \mathrm{ml}$ bacterial DNA extract and controls were amplified with $0.5 \mathrm{mM}$ primers (Forward: 5'-AGTTTGATCCTGGCTCAG -3 ' and Reverse: 5'-AGGCCCGGGAACGTATT CAC-3') (1343 bp), $200 \mathrm{mM}$ of each dNTP (Fermentas, Germany), $2 \mathrm{mM} \mathrm{MgCl} 2,10 \mathrm{mM} \mathrm{KCl}$ PCR buffer and $1.0 \mathrm{U}$ Taq polymerase (Fermentas, Germany). The DNA was amplified in a programmable thermal cycler (Eppendorf, Mastercycler ${ }^{\circledR}$ 5330, Eppendorf-Netheler-Hinz $\mathrm{GmbH}$, Hamburg, Germany) PCR device using the following protocol: $94{ }^{\circ} \mathrm{C}$ for $5 \mathrm{~min}, 40$ cycles of $94{ }^{\circ} \mathrm{C}$ for $1 \mathrm{~min}, 55^{\circ} \mathrm{C}$ for $1 \mathrm{~min}, 72{ }^{\circ} \mathrm{C}$ for 2 min, and final $72^{\circ} \mathrm{C}$ for $5 \mathrm{~min}$. Amplified products were stained with SYBR DNA gel stain, separated electrophoretically in $1.5 \%$ agarose gels, and imaged using an UV transilluminator and digital capture system. E. coli ATCC 8739 and sterile distilled water were used as a positive and negative controls, respectively.

\section{Antimicrobial susceptibility testing}

Antimicrobial resistance pattern was performed by the Kirby-Bauer disc diffusion method using Mueller-Hinton agar (HiMedia Laboratories, Mumbai, India, MV1084), according to the Clinical and Laboratory Standards Institute 
(CLSI) guidelines [17]. After incubating the inoculated plate aerobically at $37^{\circ} \mathrm{C}$ for $18-24 \mathrm{~h}$ in an aerobic atmosphere, the susceptibility of the $E$. coli isolates against tetracycline (30 $\mu \mathrm{g} /$ disk), cefotaxime (30 $\mu \mathrm{g} /$ disk), gentamycin (10 $\mu \mathrm{g} /$ disk), ampicillin (10 $\mu /$ disk), amikacin (30 $\mu /$ disk), imipenem (30 $\mu /$ disk), mezlocillin (30 $\mu /$ disk), ciprofloxacin (5 $\mu \mathrm{g} /$ disk), cotrimoxazole (30 $\mu \mathrm{g} /$ disk), norfloxacin (30 $\mu \mathrm{g} / \mathrm{disk})$, ofloxacin (5 $\mu \mathrm{g} /$ disk), nitrofurantoin (300 $\mu \mathrm{g} / \mathrm{disk})$, nalidixic acid (30 $\mu \mathrm{g} / \mathrm{disk})$, cephalothin (30 $\mu \mathrm{g} / \mathrm{disk})$ and clindamycin $(2 \mu \mathrm{g} /$ disk $)$ antimicrobial agents was measured. Results were interpreted in accordance with interpretative criteria provided by CLSI (2012). E. coli ATCC 8739 was used as quality control organisms in antimicrobial susceptibility determination.

\section{Statistical analysis}

The data were analyzed using SPSS software (version 17, SPSS Inc, United States) using Chisquare and Fisher exact tests to find any significant correlation between incidence of $E$. coli strains and their antibiotic resistance properties in pregnant women. Differences were considered significant at $p<0.05$.

\section{RESULTS}

Table 1 shows the total prevalence of $E$. coli in pregnant women with RUTIs and healthy pregnant women. All of the E. coli isolates were authenticated by the PCR amplification. Figure 1 represents the results of gel electrophoresis for E. coli bacterium. We found that 28 out of 445 $(6.29 \%)$ swab samples were positive for E. coli. On the other hand, 18 out of $200(9 \%)$ swab samples from pregnant women with history of RUTIs, while 10 out of 245 (4.08 \%) swab samples from healthy pregnant women were positive for $E$. coli. Pregnant women older than 40 years had the highest prevalence of $E$. coli $(12.71 \%)$. Statistical significant differences were seen between the types of samples and prevalence of E. coli $(p<0.01)$ and between the age of women and prevalence of E. coli $(p<$ 0.05).

Table 2 indicates the antimicrobial resistance pattern of $E$. coli strains isolated from pregnant women with RUTIs and healthy pregnant women. E. coli strains from our investigation harbored the highest levels of resistance against ampicillin (96.42\%), tetracycline $(85.71 \%)$, amikacin (71.42\%), ciprofloxacin (67.85\%) and gentamycin $(58.71 \%)$ antibiotic agents. Levels of antibiotic resistances in pregnant women with history of RUTIs were higher than those of healthy pregnant women $(p<0.05)$. The most effective antibiotic agents against $E$. coli strains from the high vaginal swab samples of pregnant women were imipenem (3.57\%), mezlocillin (25 $\%)$ and nitrofurantoin (25\%).

\section{DISCUSSION}

The results of present study are the first prevalence report of the UPEC strains and their antibiotic resistance pattern in the swab samples taken directly from the high vaginal swab samples of pregnant women. Probably the pregnant women of our study had vaginitis due to the presence of UPEC strains. History of RUTIs can accentuate the above hypothesis. It is commonly accepted that a high frequency of UTI during pregnancy would be due to physiological changes the human body undergoes during pregnancy. Bacterial vaginosis has been reported as the most common vaginal infection. Its prevalence varies from 10 to $65 \%$ [10]. Many serious obstetric and gynecological complications have been associated with bacterial vaginosis. Such obstetric complications included premature rupture of membranes, preterm labor and delivery, endometritis and chorioamnionitis [18].

Table 1: Prevalence of Escherichia coli in pregnant women with RUTIs and healthy ones

\begin{tabular}{lccc}
\hline Sample & & No. samples collected & Escherichia coli-positive (\%) \\
\hline Pregnant women & $<20$ years & 70 & $3(4.28)$ \\
with history of & $20-40$ years & 80 & $6(7.5)$ \\
RUTls & $>40$ years & 50 & $9(18)$ \\
& Total & 200 & $18(9)$ \\
Healthy pregnant & $<20$ years & 82 & $1(1.21)$ \\
women & $20-40$ years & 95 & $3(3.15)$ \\
& $>40$ years & 68 & $6(8.82)$ \\
& Total & 245 & $10(4.08)$ \\
Total & $<20$ years & 152 & $4(2.63)$ \\
& $20-40$ years & 175 & $9(5.14)$ \\
& $>40$ years & 118 & $15(12.71)$ \\
& Total & 445 & $28(6.29)$ \\
\hline
\end{tabular}


Table 2: Antimicrobial resistance pattern of Escherichia coli isolated from pregnant women with RUTIs and healthy ones

\begin{tabular}{lccc}
\hline \multirow{2}{*}{$\begin{array}{l}\text { Antimicrobial } \\
\text { agent }\end{array}$} & \multicolumn{3}{c}{ Type of samples (no. positive) } \\
\cline { 2 - 4 } & $\begin{array}{c}\text { Pregnant women } \\
\text { with history of } \\
\text { RUTIs (18) }\end{array}$ & $\begin{array}{c}\text { Healthy pregnant } \\
\text { women (10) }\end{array}$ & $\begin{array}{c}\text { Total } \\
\text { (N=28, \%) }\end{array}$ \\
\hline Tetracycline & $17(94.44)$ & $7(70)$ & $24(85.71)$ \\
Cefotaxime & $8(44.44)$ & $2(20)$ & $10(35.71)$ \\
Gentamycin & $17(94.44)$ & $7(70)$ & $24(58.71)$ \\
Ampicillin & $18(100)$ & $9(90)$ & $27(96.42)$ \\
Amikacin & $15(83.33)$ & $5(50)$ & $20(71.42)$ \\
Imipenem & $1(5.55)$ & - & $1(3.57)$ \\
Mezlocillin & $5(27.77)$ & $2(20)$ & $7(25)$ \\
Ciprofloxacin & $14(77.77)$ & $5(50)$ & $19(67.85)$ \\
Cotrimoxazole & $7(38.88)$ & $3(30)$ & $10(35.71)$ \\
Norfloxacin & $8(44.44)$ & $2(20)$ & $10(35.71)$ \\
Ofloxacin & $7(38.88)$ & $2(20)$ & $9(32.14)$ \\
Nitrofurantoin & $6(33.33)$ & $1(10)$ & $7(25)$ \\
Nalidixic acid & $7(38.88)$ & $1(10)$ & $8(28.57)$ \\
Cephalothin & $9(50)$ & $3(30)$ & $12(42.85)$ \\
Clindamycin & $10(55.55)$ & $2(20)$ & $12(42.85)$ \\
\hline
\end{tabular}

We found that the prevalence of UPEC strains in the swab samples of pregnant women with history of RUTIs and in healthy pregnant women were 9 and $4.08 \%$, respectively. Transmission of pathogenic strains of $E$. coli from the urinary tract to the vagina could be the main factor for the higher prevalence of UPEC strains in the high vaginal swab samples of pregnant women with history of RUTIs. The observed prevalence of UPEC strains in the high vaginal swab samples of healthy pregnant women could also be due to the fact that they may have been infected with UTIs in the past or even vaginitis which may been forgotten or become asymptomatic. The results of our investigation revealed that pregnancy in older ages could be the main risk factors in the transmission of infections from urinary into the reproductive system.

Our study also focused on the antibiotic resistance pattern of UPEC strains isolated from pregnant women. As a result of high and irregular prescription of antibiotic coupled with high levels of UPEC resistance against commonly used antibiotics, it was not surprising that most of the UPEC strains of our study were resistant against ampicillin, tetracycline, amikacin, ciprofloxacin, gentamycin, cotrimoxazole, norfloxacin, ofloxacin, nitrofurantoin, cephalothin and clindamycin. This observation has been corroborated by some literature reports. Barati et al [19] revealed that urine samples of pregnant women were contaminated with $E$. coli strains. They showed that the sensitivity of $E$. coli isolates against antibiotics were coamoxiclav (5.72\%), ampicillin $(8.86 \%)$, amoxicillin $(11.87 \%)$, cefazolin $(32.12$ $\%)$, cephalexin $(36.1 \%)$, gentamicin $(40.28 \%)$, cotrimoxazole (48.15\%), nalidixic acid $(55.3 \%)$, nitrofurantoin $(72.48 \%)$ and ceftriaxone $(80.78$ $\%$ ) which was similar to our results. Prevalence of UPEC strains in pregnant women in Sweden, Uganda and Vietnam during first, second and third trimester of pregnancy were 22,36 and 42 ; 9,68 and $23 \%$ and finally 12,40 and $48 \%$, respectively [20]. Guiral et al [21] in a study which was conducted on prevalence of $E$. coli in the genital tracts of the pregnant women, reported 86 out of 648 samples (13\%) which was higher than our results. High prevalence of UPEC strains in the vaginal samples of pregnant women have also been reported previously [2224]. Prevalence of antibiotic resistance in the $E$. coli strains isolated from pregnant women of Tanzania against ampicillin, sulfamethazoletrimethoprim, tetracycline, nitrofurantoin, ceftriaxone, gentamycin, ciprofloxacin and imipenem were 52.9, 64.7, 58.8, 5.9, 29.4, 5.9, 11.8 and $0 \%$, respectively [25]. Similar results have been reported from Nigeria [25], Iraq [27] and Argentina [28].

\section{CONCLUSION}

As far as we know, the present study is the first prevalence report on antibiotic resistance pattern of UPEC strains in pregnant women with history of RUTIs. The findings indicate that RUTIs and pregnancy in old age may be the two most important risk factors for the transmission of UPEC strains from the urinary tract to the vagina. Due to the clinical importance of the presence of resistant UPEC strains in upper part of the vaginal of pregnant women, accurate identification of these women and their primary treatment with imipenem, mezlocillin and nitrofurantoin may be effective in the 
management and prevention of reproductive complications. Further studies are required to evaluate the exact role of UPEC strains in pregnant women.

\section{DECLARATIONS}

\section{Acknowledgement}

The cooperation of the participants in this study is greatly acknowledged.

\section{Conflict of Interest}

No conflict of interest associated with this work.

\section{Contribution of Authors}

The authors declare that this work was done by the authors named in this article and all liabilities pertaining to claims relating to the content of this article will be borne by them.

\section{REFERENCES}

1. Ovalle A, Levancini $M$. Urinary tract infections in pregnancy. Curr Opin Urol 2001; 11(1): 55-59.

2. Chang SL, Shortliffe $L D$. Pediatric urinary tract infections. Pediatr Clin North Am 2006; 53: 379-400.

3. Mashayekhi F, Moghny M, Faramarzpoor M, Yahaghi E, Khodaverdi Darian E, Tarhriz V. Dormanesh B. Molecular Characterization and Antimicrobial Resistance of Uropathogenic Escherichia Coli. Iran J Biotech 2014; 12(2): e16833.

4. Dormanesh B, Safarpoor Dehkordi F, Hosseini S, Momtaz H, Mirnejad R, Hoseini MJ, Yahaghi E, Tarhriz $V$, Khodaverdi Darian E. Virulence factors and oserogroups profiles of uropathogenic Escherichia coli isolated from Iranian pediatric patients. Iran Red Crescent Med J 2014; 16(2): e14627.

5. Kucheria R, Dasgupta $P$, Sacks SH, Khan MS, Sheerin NS. Urinary tract infections: new insights into a common problem. Postgrad Med.J.2005; 81: 83-86.

6. Shaikh N, Morone NE, Bost JE, Farrell MH. Prevalence of urinary tract infection in childhood: a meta-analysis. Pediatr Infect Dis J. 2008; 27(4): 302-308.

7. Sobczyk D, Krynicki T, Blumczyński A, Zaniew M, Kroll P, Siwińska A, Zachwieja J. New, successful treatment of urinary tract infection caused by Pseudomonas aeruginosa Przegl Lek. 2006; 63 Suppl 3: 140-141.

8. Totsika M, Moriel DG, Idris A, Rogers BA, Wurpel DJ, Phan MD, Paterson DL, Schembri MA. Uropathogenic Escherichia coli mediated urinary tract infection. Curr Drug Targets 2012; 13(11): 1386-1399.

9. Hooton TM, Fihn SD, Johnson C, Roberts PL, Stamm WE. Association between bacterial vaginosis and acute cystitis in women using diaphragms. Arch Intern Med 1989; 149(9): 1932-6.
10. Sumati AH, Saritha NK. Association of urinary tract infection in women with bacterial vaginosis. J. Glob. Infect. Dis 2009; 1(2): 151-152.

11. Hillerbrand L, Harmanli $\mathrm{OH}$, Whiteman V. Urinary tract infections in pregnant women with bacterial vaginosis. Am J Obstet Gynecol 2002; 186: 916-917.

12. Afrakhtech $M$, Mahdavi A. Bacterial vaginosis and urinary tract infection J. Obstet Gynecol India 2007; 57: 513516.

13. Jadhav S, Hussain A, Devi S, Kumar A, Parveen $S$, Gandham N, et al. Virulence characteristics genetic affinities of multiple drug resistant uropathogenic Escherichia coli from a semi urban locality in India. Plos One 2011; 6: e18063.

14. Asadi S, Kargar M, Solhjoo K, Najafi A, Ghorbani-Dalini $S$. The association of virulence determinants of Uropathogenic Escherichia coli with antibiotic resistance. Jundishapur J Microbiol 2014; 7: e9936.

15. Ethics and research on human subjects: International guidelines. J Med Ethics. 1995; 21(6): 363-364. PMCID: PMC1376838.

16. Ekhaise FO, Richard FR. Common Bacterial Isolates associated with Semen of Men Complaining of Infertility in University of Benin Teaching Hospital (U.B.T.H), Benin City, Nigeria. World J Med Sci 2008; 3: 28-33.

17. Clinical and Laboratory Standards Institute (CLSI). Performance Standards for Antimicrobial Disk Susceptibility Tests, Approved Standard-Ninth Edition (M2-A9). United States: Clinical and Laboratory Standards Institute; 2012.

18. Harmanli $\mathrm{OH}$, Cheng $G$, Nyirjesy $P$, Chatwani $A$, Gaughan JP. Urinary tract infections in women with bacterial vaginosis. Obstet Gynecol 2000; 95: 710-2.

19. Barati L, Ghezelsofla F, Azarhoush R, Heidari F, Noora M. Antibiotic sensitivity of isolated E.coli from pregnant women urine. J Gorgan Uni. Med. Sci. 2011; 13(3): 101107.

20. Ramos NL, Sekikubo M, Dzung DT, Kosnopfel C, Kironde F, Mirembe F, Brauner A. Uropathogenic Escherichia coli isolates from pregnant women in different countries. J Clin Microbiol 2012; 50(11): 35693574.

21. Guiral E, Bosch J, Vila J, Soto SM. Prevalence of Escherichia coli among samples collected from the genital tract in pregnant and non-pregnant women: relationship with virulence. FEMS Microbiol Lett 2011; 314(2): 170-173.

22. Cook SW, Hammill HA, Hull RA. Virulence factors of Escherichia coli isolated from female reproductive tract infections and neonatal sepsis. Infect Dis Obstet Gynecol 2001; 9: 203-207.

23. Birosová E, Siegfried L, Kmet'ová M, Makara A, Ostró $A$, Gresová A, Urdzík P, Liptáková A, Molokácová M, Bártl $R$, Valanský $L$. Detection of virulence factors in alphahaemolytic Escherichia coli strains isolated from various clinical materials. Clin Microbiol Infect 2004; 10(6): 569573 
24. Obata-Yasuoka M, Ba-Thein W, Tsukamoto $T$, Yoshikawa H, Hayashi H. Vaginal Escherichia coli share common virulence factor profiles, serotypes and phylogeny with other extraintestinal E. coli. Microbiol. 2002; 148: 2745-2752.

25. Masinde A, Gumodoka B, Kilonzo A, Mshana SE. Prevalence of urinary tract infection among pregnant women at Bugando Medical Centre, Mwanza, Tanzania. Tanzania J. Health Res. 2009; 11: 154-161.

26. Olufunke OA, Abiodun AO, Dunah FC. Extended Spectrum Beta- Lactamase- Producing Uropathogenic
Escherichia coli in Pregnant Women Diagnosed With Urinary Tract Infections in South-Western Nigeria. J. Mol. Biol. Res. 2014; 4: 34-41.

27. Al-Mayahie SMG. Phenotypic and genotypic comparison of ESBL production by Vaginal Escherichia coli isolates from pregnant and non-pregnant women. An. Clin. Microbiol. Antimicrob. 2013; 12: 7-11.

28. Villar HE, Aubert V, Baserni MN, Jugo MB. Maternal carriage of Extended-Spectrum Beta-Lactamaseproducing Escherichia coli isolates in Argentina. J Chemother 2013; 25(6): 324-327. 